\title{
Analysis and Research on Broken-off Driving Bevel Gear shaft of Automobile Axle

\author{
Zhanlong Cao
}

School of Mechanical Engineering,Hubei University of Automotive Technology,Shiyan City,Hubei 442002, P.R.China

*caozl@mail.ustc.edu.cn

Keywords: automobile axle; driving bevel gear; broken; annealing

\begin{abstract}
Based on the phenomenon of broken in the running process of the drive Gear shaft of Automobile Axle. It was found that the major site of broken for thread and spline connected region. The process of the gear shaft and the shaft material of the broken and the structure of thread and spline connected region were analyzed. Finally, it was found that the annealing time of thread and the structure of thread and spline connected region have some problems. The structure of thread and spline connected region was optimized. After improvement, it was not occurred the phenomenon of broken in the running process of the drive Gear shaft of Automobile Axle.
\end{abstract}

\section{Introduction}

Automobile axle is connected with the vehicle frame by a suspension, the wheel installed on both ends. its role is to bear the load and maintain normal driving automotive vehicle on the road. For trucks, the bridge is a core component of truck chassis dynamics system, which determines the quality of automotive vehicle safety and reliability. Main reducer is a major component of truck axles, and driving bevel gear shaft is the main key parts reducer. Therefore, the driving bevel gear shaft break, will cause adverse effects. The reason for broken of driving bevel gear shaft of automobile axle is analyzed. Effective solutions are proposed, which help to avoid the occurrence of such failures.

\section{Analysis for broken of driving bevel gear shaft}

Introduction of broken of driving bevel gear shaft. From one year of axle fault analysis found that driving bevel gear axle of the main reducer of shaft broken occurred during operation process. The number of broken is up to 88 in a year. Number winter break member is 71 , the other three quarters the number of broken is 16. It was found that the major site of broken for thread and spline connected region. As shown in figure 1. Thus, the reason for the fault to be analyzed, thereby is eliminating the fault.
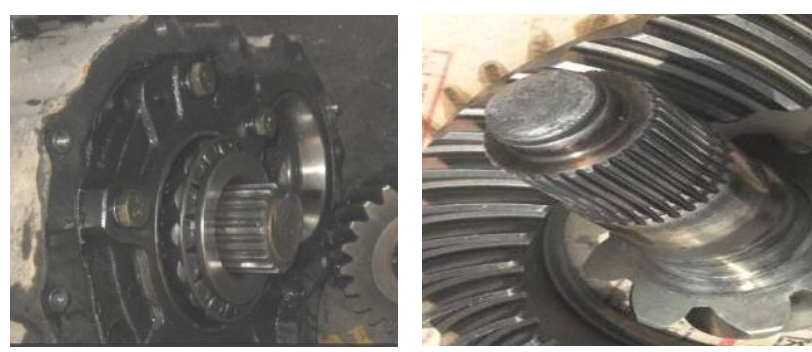

Figure1 failure of the driving bevel gear shaft

Machining process analysis of driving bevel gear shaft. The main processing technology of the main processing technology of the driving bevel gear shaft is the following material, forging forming, pre heat treatment, machining, carburizing and quenching, tempering, annealing, finishing, matching, and so on. While the driving bevel gear shaft screw part in order to meet the requirements in the assembly and working state, the need for low hardness, so it should be carried out annealing, local medium frequency annealing. Statistics found by the larger number of driving bevel gear shaft fracture 
in winter, when setting the annealing time Seasons power and power is the same, but compared to the other quarters winter indoor temperature is low, equivalent to shortening the annealing time and power, will lead thread of high hardness, which occurred in breakage. 10 samples were taken. Driving bevel gear shaft thread and other parts of the winter season production of hardness are compared. As shown in figure 2. Seen from the figure, the universal hardness winter than in other seasons hardness was found to be higher hardness values higher 1-4HRC. Set the same annealing time in four seasons, and power was unreasonable. Reasonable season annealing time and power is the need to set.

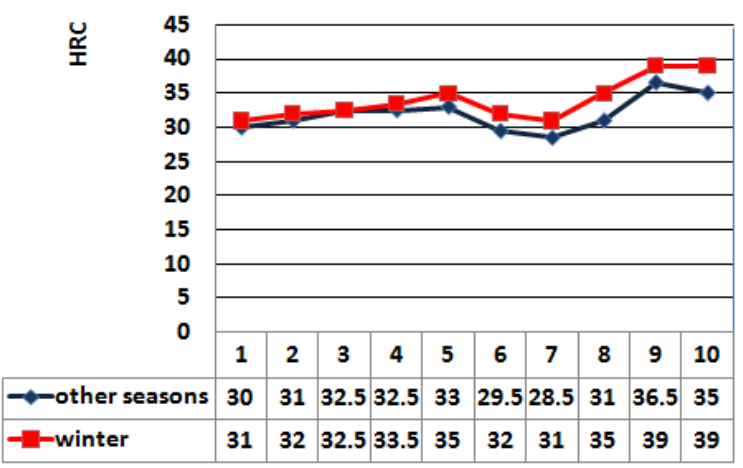

Figure2 Comparison Chart driving bevel gear shaft thread parts, winter and other seasons hardness

Analysis of material the broken driving bevel gear shaft. $20 \mathrm{CrMnTiH}$ is selected in driving bevel gear shaft material, after heat treatment requirements for the effective hardness layer $1.5-2 \mathrm{~mm}$, surface hardness layer requirements 59-63HRC, core hardness requirements 30-40HRC. Fracture failure of the sample is extracted and carried out analysis of the material, samples effective hardness layer deep $1.6 \mathrm{~mm}$, surface hardness of $60 \mathrm{HRC}$, hardness $35 \mathrm{HRC}$, the surface layer of martensite and residual austenite 2-3 2 level, the center organized a very small amount of lath martensite and ferrite. As shown in figure 3. Test results show that the samples meet the requirements, the material is no problem.

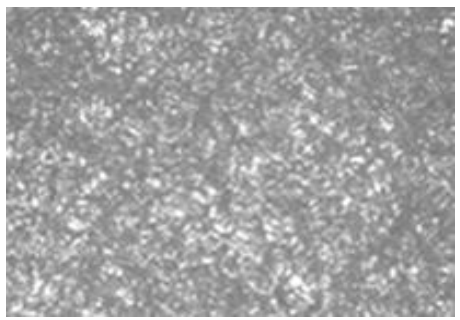

Surface tissue

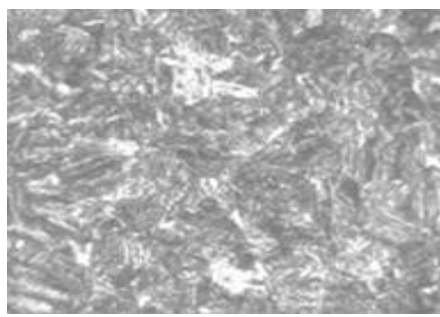

Heart tissue

Figure 3 Microstructure Figure driving bevel gear shaft

Structural analysis of the driving bevel gear shaft. The main structure of the thread, spline gear is a driving bevel gear shaft. As shown in figure 4.

The figure can be seen through the threaded portion and the spline area in a transitional structure, size and thread diameter relatively close, there is no obvious transition stage, the threaded portion of the spline-sectional transition that much difference, transition mode is unreasonable, excessive stress Assembly lead to driving bevel gear shaft breakage or damage. Therefore, the structure here must also be improved and optimized.

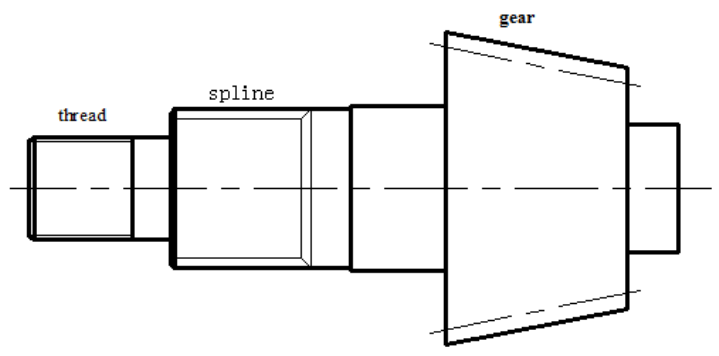

Figure4 Structural of the driving bevel gear 
Summary of broken Analysis of driving bevel gear shaft .Driving bevel gear shaft fracture failure process analysis pieces, materials analysis, structural analysis was analyzed and found that the annealing time and annealing process is leading to the same power Seasons driving bevel gear shaft breakage or damage for a reason; Driving bevel gear shaft breaking material to be analyzed, the analysis shows compliance; for driving bevel gear shaft structure is analyzed, and found the threaded portion and the spline area there is a significant transition in the structure, local stress too large, the driving bevel gear shaft fracture or destruction. Therefore, it is necessary to take the initiative annealing process and structure of the bevel gear shaft improvements and optimization.

\section{Optimum Design of driving bevel gear shaft}

Annealing process control. Different annealing time and annealing power is designed in the driving bevel gear shaft threaded parts, in order to eliminate the winter and other seasons of hardness differences. By increasing the annealing time or increase the annealing power to eliminate the differences in a way, it needs to find suitable annealing process parameters through the test. Hardness values in other seasons 60s annealing time and annealing hardness values $36 \mathrm{Kw}$ power is referenced standard seat test,and measured the threaded portion 5 annealing of this sample after process parameters. Test packets are shown in Table 1.

Table 1 grouping table annealing process parameters

\begin{tabular}{ccc}
\hline $\begin{array}{c}\text { Seria } \\
1 \\
\text { numbe } \\
r\end{array}$ & Power $(\mathrm{Kw})$ & \\
\hline 1 & 36 & Annealing time $(\mathrm{s})$ \\
2 & 36 & 65 \\
3 & 36 & 70 \\
4 & 37 & 75 \\
5 & 38 & 60 \\
6 & 39 & 60 \\
\end{tabular}

experimental groups,

Through the above and each group of threaded portion of the test measured hardness values after annealing, As shown in figure 5. As can be seen from the figure, the annealing power increase and annealing time longer, threaded parts after annealing hardness value will decrease. Compare annealing power $36 \mathrm{Kw}$ and annealing time standard hardness $60 \mathrm{~s}$ when, from measured values and fitting trend line, annealing power of $36 \mathrm{Kw}$ and annealing time hardness value and standard hardness values closest $70 \mathrm{~s}$. Annealing power of $36 \mathrm{Kw}$ and annealing time is 70 s annealing process parameters are adopted in winter.

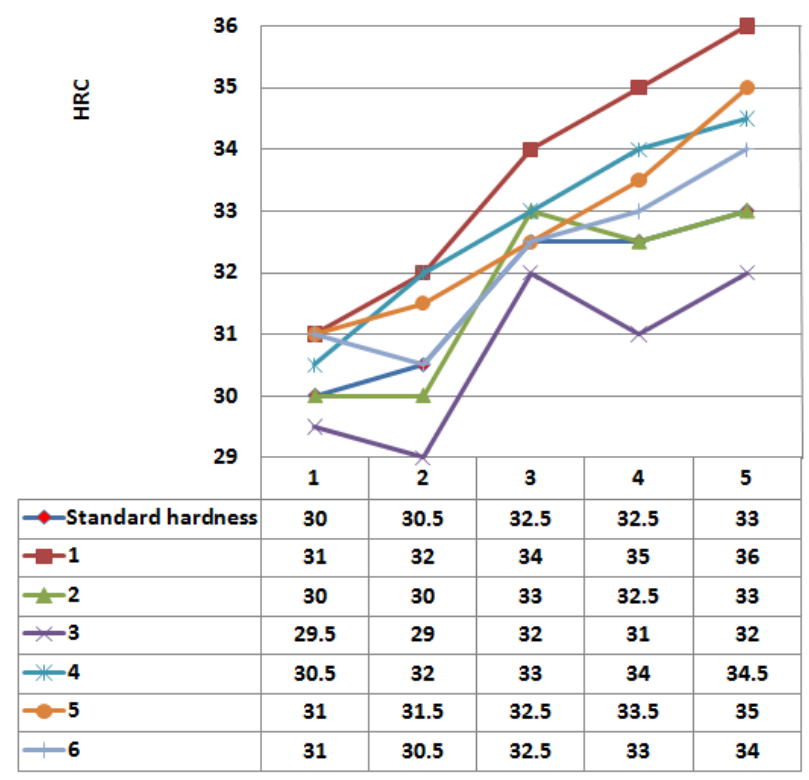


Figure 5 test results

Structure optimization of driving bevel gear shaft. Increasing the transition section method is adopted in the driving bevel gear shaft thread design area and spline connected. The gear shaft threads and splines stepped transition interval increases, the size of the original diameter 46 into a diameter of 54. As shown in figure 6.

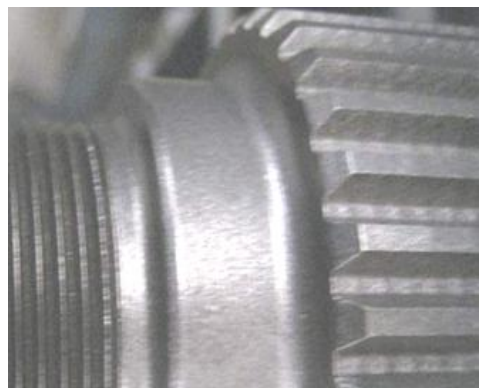

Improvement

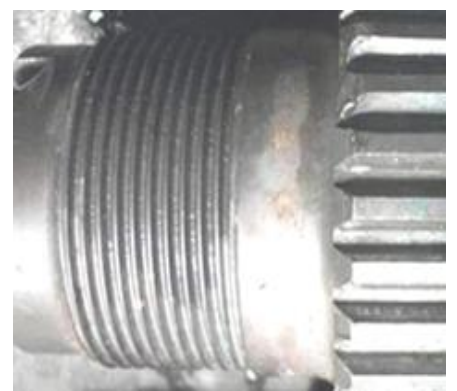

not improvement

Figure6 improved contrast for the structure of connected to threaded portion and the spline

\section{Summary}

Driving bevel gear shaft broken failure was analyzed. threaded parts are set to the same annealing time and annealing power have a problem during the winter and other seasons. There was a problem connected with the spline threaded structure transition region also. Therefore, the annealing process parameters and thread and spline connected to the regional structure was improved. Annealing process parameters were set in winter. Spline connected to the threaded area was designed transition structure. After these improvements, Automobile axle run was tracked, the broken of driving bevel cear shaft of automobile axle does not reappear.

\section{References}

[1] Fan Yang,Hongdong Ren,Shouwei,An Oil Temperature Analysis \& Control of Axle Drive Head, Automobile Parts. 2013(1)82-85.

[2] Feng Yang .Fracture failure analysis of motor gear, Heat Treatment of Metals.2014(6)155-157

[3] Lianguang Zhu, Xiangdong Feng, Kewei Song, Qingjian Li. Study and Application of Local Softening Process on Driving Bevel Gear of STR Heavy-DutyTruck, Heat Treatment of Metals.2005(4)52--54

[4] Yong Huang,Jianqiang Xiao,Failure Analysis and its Control Method of Fracture For Threaded Parts after Annealing, Heat Treatment Technology and Equipment. 2010(12)7-10.

[5] Mingxia Hu,Improvement on Heat Treatment Process of Driving Bevel Gear, Heat Treatment of Metals.2001(12)46-47 